\title{
Static and dynamic characterization of pull-in protected CMOS compatible poly-SiGe grating light valves
}

\author{
S. Rudra ${ }^{\text {a }}$, J. De Coster ${ }^{\text {b }}$, R. Van Hoof ${ }^{\text {b }}$, G. Bryce ${ }^{\text {b }}$, S. Severi ${ }^{\text {b }}$, A. Witvrouw ${ }^{\text {b }}$, D. Van \\ Thourhout ${ }^{\text {a }}$ \\ ${ }^{\text {a }}$ Photonics Research Group, INTEC, Ghent University- IMEC, Gent, Belgium \\ ${ }^{\mathrm{b}}$ IMEC, Leuven, Belgium
}

Grating Light Valve (GLV) display pixels are reflection type diffraction gratings consisting of electrostatically movable coplanar microbeams. Once actuated, the alternate movable beams deflect downwards which produces controlled diffraction of light creating bright and dark pixels in a display system. GLV displays provide a huge improvement in contrast ratio and resolution over other MOEMS devices. At the same time, compared to hybrid integration, post processing of MEMS monolithically on top of CMOS can lead to increased functionality, performance and reliability. Poly-SiGe structural layers can be deposited at low temperature $\left(\sim 450^{\circ} \mathrm{C}\right)$, allowing to retain the performance of underlying CMOS electronics though possessing the desired material properties for MEMS. Hence the aim of this work is to fabricate CMOS compatible poly-SiGe GLVs and to study their static and dynamic behavior. A novel process flow was developed regarding the deposition of thin poly-SiGe structures which is well within the maximum thermal range to retain the full functionality of the underlying CMOS circuitry. A contrast of over 1500:1 was obtained showing excellent optical response of the devices. The effect of squeeze film damping in determining the dynamic response of the GLVs is thoroughly investigated. Influence of variation in dimensional parameters on the settling time of the structures is discussed in detail. A minimum settling time of $2 \mu s$ was achieved for our devices. We also showed the analog gray scale nature of the GLVs. In addition, we also use the technique of mechanical stoppers to avoid accidental destruction of the devices because of the pull-in phenomenon.

Keywords: Grating, Poly-SiGe, Squeezed film damping

\section{Introduction:}

The Grating Light Valve [1, 2] (GLV) is a microelectromechanical reflection grating. It consists of clampedclamped beams suspended in air over a conducting substrate with the help of anchors on the two far ends allowing them to move vertically. The microbeams are held in tension so that when non-actuated they remain flat and form a reflective surface (OFF state). The alternate microbeams of the GLVs can be actuated over a wide range of levels in a large 2D array and hence a quasi-continuous phase function can be imprinted onto the incoming light beam (ON state). The result is an entirely reconfigurable diffractive element in reflection mode providing precise and accurate pixel-by-pixel brightness control. This key feature of GLVs enables the realization of smooth gradation control and a high contrast ratio resulting in rich and detailed images. A detailed description of the operational principles of the GLVs can be found elsewhere [2, 3].

At the same time, integration of MEMS devices with the electronic circuits is becoming increasingly important for compactness and performance reasons [4]. In hybrid integration, separate use of MEMS and the electronic circuits results in performance-limiting parasitics arising mainly from the size of the bondpads and from the long bonding wires. Comparatively, monolithic integration of MEMS with CMOS can improve performance, yield and reliability as well as lower the manufacturing, packaging and instrumentation costs [5]. Poly-SiGe (deposition temperature $\sim 450^{\circ} \mathrm{C}$ ) has been demonstrated to be an 
ideal material for a MEMS-last monolithic processing, since films with very good electrical and mechanical properties can be obtained at CMOS-compatible temperatures [6]. This makes poly-SiGe to be very well suited for applications that need large arrays of MEMS devices, which need to be individually connected to interfacing circuits [7] (such as micro-mirror arrays, bolometers or other imaging applications).

Hence in this work we developed a new poly-SiGe process flow with relatively thin structural layers (300 $\mathrm{nm}-350 \mathrm{~nm}$ ) which is well below the range of maximum thermal load to deteriorate the underlying CMOS functionality. We used this novel process flow to develop GLVs and characterized their overall optical and mechanical performances. We were able to produce sufficient tensile stress within the poly-SiGe microbeams resulting in flat structures, one of the primary requirements for a GLV to work efficiently. We showed the variation in the amount of damping with different dimensions of the microbeams and how the resulting switching time of the individual pixels is affected. We used mechanical stoppers [8] to avoid irreversible damage when the device is accidentally pulled-in [9].

\section{Theory:}

According to scalar diffraction theory [10], the diffraction angle $\theta_{\mathrm{m}}$ for different diffraction orders $\mathrm{m}$ resulting from a grating with period $\Lambda$ is defined as:

$\sin \theta_{m}=\sin \theta_{i}+m \lambda / \Lambda$

where, $\theta_{\mathrm{i}}$ is the angle of incidence and $\lambda$ is the wavelength.

Given the high fill factor of GLVs, they can be approximated as a binary diffraction grating [11] with changing phase due to the controlled displacement of the alternate microbeams. The intensity of the light diffracted in different orders $(\mathrm{m})$ for a binary grating with normal incidence is given by:

$$
I_{m}=I_{i} \sin ^{2}(\Theta / 2) \operatorname{sinc}^{2}(m \pi / 2)
$$

Where $l_{i}$ is the intensity of the incident light and $\Theta$ is the grating phase.

For normal incidence, $\Theta$ is defined as: $\Theta=4 \pi h / \lambda$

with $h$ is the grating depth.

From the above equations, it is clear that the highest intensity for the $1^{\text {st }}$ order diffracted light is obtained when $\mathrm{h}=\lambda / 4$. In that case, the maximum diffraction efficiency in the $\pm 1^{\text {st }}$ orders will be $\sim 81 \%$ (considering $100 \%$ fill factor), whereas, for all the even orders there will be no diffracted light.

Another important parameter for the GLVs is the resonance frequency which primarily determines its fastest achievable modulation rate. As the GLVs consist of clamped-clamped type microbeams with tensile stress, the spring constant $(k)$ and the natural vibration frequency $\left(\omega_{r}\right)$ are given by $[12,13]$ :

$k=32 E w(t / l)^{3}+8 \sigma(1-v) w(t / l)$

$\omega_{r}=\frac{\alpha^{2}}{\sqrt{12}} \frac{t}{l^{2}}\left(\frac{E}{\rho\left(1-v^{2}\right)}\right)^{0.5}\left(1+\gamma\left(\frac{\sigma\left(1-v^{2}\right)}{E}\right) \frac{l^{2}}{t^{2}}\right)^{0.5}$

where, $E$ is the Young's modulus, $\rho$ is the specific mass, $v$ is the Poisson's ratio, $\sigma$ is the tensile stress, $w$ is the width, $t$ is the thickness, $l$ is the length and $m$ is the mass of the microbeams with $\alpha=4.73$ and $y=$ 0.295 [13]. It is clear from the above equations that though the resonance frequency is independent of the width, it is strongly dependent on the length and thickness of the microbeams. At the same time, the higher the tensile stress within the structures, the higher will be the modulation rate of the devices. 
Additionally, for MEMS devices, a large part of the device dynamics is determined by air damping [14]. Proper understanding of the effect of air damping for GLVs is highly necessary to optimize their dynamic response. Due to the small airgap between the microbeams and the underlying substrate and due to the fast operating speed of the GLVs, squeeze film damping $[15,16]$ becomes the dominant mechanism affecting the dynamic response of the devices. As the GLVs can be approximated by a mass-springdamper model, viscous drag due to the surrounding fluid [17] becomes the dominant damping factor in these structures. The viscous damping coefficient $c_{v}$, for parallel plate movement is given by:

$c_{v} \propto \mu l w^{3} / h_{0}^{3}$

where, $\mu$ is the viscous coefficient of the surrounding fluid and $h_{0}$ is the height of the air gap for the devices.

Hence the damping ratio of the system is defined as:

$b=c_{v} / 2 m \omega_{r}$

If the damping is too low then the resonance effects result into ringing of the microbeams when excited with a step voltage; on the other hand, too large damping also degrades the switching time of the devices. Here we show how the different parameters of the microbeams (length, width, thickness and airgap) can be varied to optimize the damping in the structures to obtain the fastest settling of the devices.

\section{Fabrication of GLVs:}

The general process flow for the poly-SiGe GLVs is schematically shown in figure 1. A standard Si wafer is used as the starting substrate. A 1 um thick High Density Plasma (HDP) Si-oxide layer is deposited over the Si-wafer followed by the deposition of a trilayer of Ti $(20 \mathrm{~nm}) / \mathrm{AlCu}(880 \mathrm{~nm}) / \mathrm{TiN}(60 \mathrm{~nm})$ after a short degas. The metal stack was subsequently patterned to define the underlying metal connections to the bondpads. A $1650 \mathrm{~nm}$ of HDP Si-oxide was added further on top of the existing structures and planarized by CMP. Next, a $200 \mathrm{~nm}$ additional HDP Si-oxide was added and the wafers were then annealed at $455^{\circ} \mathrm{C}$ for 30 mins. Later, a $400 \mathrm{~nm}$ of SiC layer was deposited on top of the planar oxide to protect the bottom dielectric layers during the aggressive vapor HF-based release process. This protection layer was subsequently patterned and plasma-etched, stopping on the underlying metal layer to define the opening for the MEMS via.

A trilayer of $\mathrm{Ti}(5 \mathrm{~nm}) / \mathrm{TiN}(10 \mathrm{~nm})$ / poly-SiGe $(380 \mathrm{~nm})$ was deposited at a maximum wafer temperature of $450^{\circ} \mathrm{C}$. This layer fills the via holes and at the same time creates the electrode layer on top of which a $250 \mathrm{~nm}$ Si-oxide hardmask is deposited and patterned. Further, an extra SiGe layer is deposited to form a thicker electrode. A single etch process was used to pattern both the thin and thick electrode layers by using the oxide hardmask as an etch stop layer. The thick electrode structures are therefore defined by lithography while the Si-oxide hardmask defines the thin electrode structures. Next, the electrode structures were planarized by the deposition of a HDP Si-oxide layer followed by CMP which makes the thick electrode to be $\sim 50 \mathrm{~nm}$ thicker than the thinner one. After this planarization with stop on the SiGe layer, the sacrificial HDP oxide layer was added on top of the electrodes to define the actuation airgap for the MEMS structures. Two different thicknesses were used, $350 \mathrm{~nm}$ and $800 \mathrm{~nm}$ - as measured from cross section SEM pictures. In this sacrificial oxide, the anchors for anchoring the structural layer to the electrode are etched. These anchors are filled during the deposition of the SiGe structural layer.

The structural layer is a B-doped poly-SiGe layer with a thickness of $330 \mathrm{~nm}(380 \mathrm{~nm})$ which is deposited by chemical vapor deposition (CVD) at a wafer temperature of $450^{\circ} \mathrm{C}$ on top of a $\mathrm{Ti}(5 \mathrm{~nm}) / \mathrm{TiN}(10 \mathrm{~nm})$ adhesion layer. The precursor gasses are $\mathrm{SiH}_{4}, \mathrm{GeH}_{4}$ and $\mathrm{B} 2 \mathrm{H} 6$. The $\mathrm{SiH}_{4}: \mathrm{GeH}_{4}$ ratio equals 0.9:1 and 
a $\mathrm{B} 2 \mathrm{H} 6(1 \%$ in $\mathrm{H} 2)$ flow of $90 \mathrm{sccm}$ was used at a wafer temperature of $450^{\circ} \mathrm{C}$ resulting in an expected Ge concentration of $78 \%$ [18]. The total processing time at $450^{\circ} \mathrm{C}$ was 12.5 minutes for a $330 \mathrm{~nm}$ thick layer and 14.2 minutes for a $380 \mathrm{~nm}$ thick layer. Next, a CMP step was used on the SiGe structural layer for roughness reduction. This CMP step also reduces the thickness of the structural layer down to $300 \mathrm{~nm}$ $(350 \mathrm{~nm})$ as observed from figure 2. Then a barrier layer of $5 \mathrm{~nm}$ thick SiC and a $30 \mathrm{~nm}$ thick Al layer were further added on top of the structural poly-SiGe layer to increase the reflectivity of the structures.

To create the bondpads, a low temperature protecting oxide was deposited on top of the structural layer stack followed by a subsequent patterning to create bondpad openings. These openings were filled with metals and later patterned to form the bondpads. Next, another protecting oxide layer was deposited on top of the bondpads. Further, the poly-SiGe structural layer was patterned to create the fixed-fixed microbeams defining the GLVs. Finally the samples were released with a 5 step release recipe in a vapor of HF and ethanol. By breaking the release process in several steps, the water formed as by-product could be efficiently removed which decreases the chance of stiction of the structural layer. Figure 3 shows the SEM picture of the top view of a fabricated GLV device.

As we later want to process these gratings on top of CMOS, it is important to investigate the thermal load during the whole process flow. For the process flow described above, the total GLV processing time at $450-455^{\circ} \mathrm{C}$ would be $<3$ hours. This total period is calculated by adding the times required for the different poly-SiGe depositions and annealing steps, as these are the operations requiring the highest temperature. This total time is significantly lower than the maximum time of 6 hours at $455^{\circ} \mathrm{C}$ which was tested for a Cu-based $0.13 \mu \mathrm{m}$ CMOS process [19] and was found not to degrade the underlying CMOS functionality. Hence, we can conclude that the GLV devices are fully compatible for processing on top of CMOS.

The strain gradient in cantilever beams was measured to be $\sim 7 \times 10^{-4} / \mu \mathrm{m}$, which makes this process also suitable for processing micro-mirrors [20]. Due to difficulties in performing stress measurements on the thin SiGe samples, the resonance frequency of fixed-fixed beams with different lengths were measured and then fitted to find the Young's Modulus $(E)$ and the tensile stress $(\sigma)$ in the SiGe structural layer. As can be seen from the figure $4, E=120 \mathrm{GPa}$ and $\sigma=20 \mathrm{MPa}$ result in a perfect fit. The maximum resonance frequency that was achieved in our structures is $1 \mathrm{MHz}$ which corresponds to the shortest microbeam of $50 \mu \mathrm{m}$.

Thus, for this work we used two different thicknesses (300 nm and $350 \mathrm{~nm}$ ) for the structural poly-SiGe layer and two different heights $(400 \mathrm{~nm}$ and $850 \mathrm{~nm})$ for the airgap. In addition, three different beam lengths of $50 \mu \mathrm{m}, 75 \mu \mathrm{m}$ and $100 \mu \mathrm{m}$ were investigated for this work each with periods of $3.0 \mu \mathrm{m}, 4.2 \mu \mathrm{m}$ and $5.0 \mu \mathrm{m}$. A fixed gap of $0.3 \mu \mathrm{m}$ among the consecutive microbeams was maintained.

\section{Results and Discussions:}

\section{- Static measurements:}

One of the stringent requirements for a GLV to function well is to achieve sufficient flatness of the microstructures. Any height difference among consecutive microbeams will result in scattering of the incident light and hence increases the noise in the dark state. For a $100 \mu \mathrm{m}$ long non-actuated grating device, with an optical profilometer we measured a height difference of $2.6 \mathrm{~nm}$ among the consecutive microbeams which indicates an excellent planarity of the fabricated devices.

\section{- Optical characterization:}

Figure 5 shows the experimental set-up used for characterization of the GLVs. The two convex lenses in the front act as a collimator. The cylindrical lens focuses the light as a horizontal line with uniform intensity 
on the center of the microbeams. The beamsplitter helps in separating the incoming and outgoing light. A photodiode in series with a slit is used to measure the intensity of the individual diffracted orders as a function of amplitude of the applied actuation voltage. We could obtain a minimum line-width of $19.5 \mu \mathrm{m}$ for the focused spot using this set-up. The length of the line can be changed by changing the opening of the front slit. We used the full 2D array of the successive microbeams to measure the contrast and efficiency of the whole device using this set-up.

Figure 6 shows the analog optical behavior of the GLVs with $400 \mathrm{~nm}$ airgap. A clear increase in the $+1^{\text {st }}$ order diffracted intensity can be observed with increasing external DC bias. When the displacement of the microbeams exceed $\mathrm{h}=\lambda / 4$, the diffraction efficiency decreases again, as expected from eq. 2 . We used a $405 \mathrm{~nm}$ violet laser to characterize the optical response of the GLVs and to estimate the maximum contrast achievable for the devices. The dark state noise obtained in the non-actuated state is much lower compared to our previous reported work [3]; mainly due to the improved flatness among consecutive microbeams. For the current devices, a maximum contrast of $\sim 1530: 1$ was obtained reproducibly showing the usefulness of our GLVs in making high quality displays.

Though theoretically the maximum achievable intensity in the $\pm 1^{\text {st }}$ order is $81 \%$ for a binary grating (eq. 2 ), considering $92 \%$ reflectivity of the top Al layer and $94 \%$ fill factor for our gratings, the maximum attainable intensity in the $1^{\text {st }}$ order turns out to be $\sim 71 \%$. For our GLVs, the maximum diffracted light measured in the first order is $\sim 67 \%$ (combining $+1^{\text {st }}$ and $-1^{\text {st }}$ orders) which follows the theoretical prediction very closely.

As the beams are curved from the anchors onwards with maximum deflection occurring only at the center, an ideal square pattern is never obtained [21]. The longer the microbeam, the flatter is the deflection around its center. A comparison of the deflected shape (as obtained from a COMSOL Multiphysics ${ }^{\text {TM }}$ simulation) of the fixed-fixed microbeams and the Gaussian intensity distribution of the focused spot is shown in figure 7 . As can be seen, the variation in height of the central deflected region of a $75 \mu \mathrm{m}$ long microbeam is much smaller than that of the $50 \mu \mathrm{m}$ long microbeam. Consequently, for the same vertical deflection, more light is diffracted with the same phase for the longer GLVs. As a result, higher diffraction efficiency and hence higher contrast is obtained for $75 \mu \mathrm{m}$ long GLVs than that of the $50 \mu \mathrm{m}$ long ones.

\section{- Effect of squeeze film damping in GLVs}

As discussed before, squeeze film damping plays an important role in the dynamic response of the devices. To take advantage of the relatively high resonance frequency, the damping in these devices should also be high enough to suppress oscillations. We used laser doppler vibrometry to characterize the response of the GLVs to a square wave pulse train and varied different dimensional parameters to determine their influence on the dynamics of the devices.

As seen from eq. 6 , the most important parameter influencing the damping of the devices is the airgap ( $c_{v}$ $\propto 1 / h_{0}{ }^{3}$ ). Fig. 8 shows the dependence of the settling time (equilibrium $\pm 2 \%$ ) of the microbeams on the underlying airgap thickness. Whereas the settling time was $~ 20 \mu \mathrm{s}$ for a microbeam with $850 \mathrm{~nm}$ airgap, it reduced to $2.2 \mu \mathrm{s}$ by changing the airgap to $400 \mathrm{~nm}$. With decreasing airgap, the underlying fluid is more efficiently trapped during displacement of the microbeams, damping the overshoots more effectively resulting in faster settling. Since the GLV beams are electrostatically actuated, their dynamic operation is inherently limited by the pull-in phenomenon (maximum deflection $=h_{0} / 3$ ). Hence to obtain the maximum diffraction efficiency, the sacrificial layer thickness should be designed in such a manner that $h_{0} / 3>\lambda / 4$. As a result, the airgap is constrained by the wavelength of operation. Further in this paper, we show an approach which is used to avoid destruction of the devices due to pull-in. In that case the airgap can be made closer to its minimum permissible value of $3 N / 4$. 
Fig. 9 shows the variation in settling time of the devices with change in width for a $50 \mu \mathrm{m}$ long microbeam with an airgap of $400 \mathrm{~nm}$. As can be seen clearly from the figure, with increasing width the mechanical oscillations and overshoots are more efficiently damped resulting in a faster settling of the devices. Whereas the settling time was $\sim 6.5 \mu$ s for a $2.7 \mu \mathrm{m}$ wide microbeam, it reduced to $2.2 \mu \mathrm{s}$ by increasing the beam width to $4.7 \mu \mathrm{m}$. But the beam width is also related to the angular separation among different diffracted orders as shown in equation 1. Hence, for a larger width, the effective separation and collection of the $0^{\text {th }}$ and the $\pm 1^{\text {st }}$ orders of diffracted light become more difficult, resulting in either a reduced contrast or a more extended optical setup.

Variation in settling time of the devices with the thicknesses of the microbeams is shown in fig. 10. The two microbeams differ only by their thickness and we actuated both of them in the same way. As can be seen from the figure, for the thicker microbeam, the amplitude of the overshoot and ringing is larger than that of the thinner one. Hence, faster settling is obtained for the thinner microbeam. But with lower thickness the resonance frequency also scales down. Hence a minimum thickness of the beam has to be chosen to achieve a resonance frequency suitable for the targeted application. Any additional thickness beyond the minimum value will degrade the settling time.

Fig. 11 shows the dependence of the settling time on the length of the microbeams. Whereas a $4.7 \mu \mathrm{m}$ wide and $50 \mu \mathrm{m}$ long beam with $400 \mathrm{~nm}$ airgap shows an under-damped nature, longer beams of $75 \mu \mathrm{m}$ and $100 \mu \mathrm{m}$ lengths with identical parameters become critically damped and over-damped respectively. It suggests that with increasing length, the damping ratio increases and accordingly the nature of damping in the system is changed as predicted from eq. 7. It is clear from figure 4 that though longer beams help in faster settling, it decreases the resonance frequency and hence the modulation rate of the devices. On the other hand, shortening the length of the beams increases the actuation voltage and also requires critical optical settings to obtain a narrower focused spot at the center of the beams. Hence a judicial choice of the length of the beams has to be made depending on the target application of the devices.

\section{- Analog gray scale of GLVs}

One of the most important advantages of GLVs over other optical MEMS is its intrinsic analog capability of producing gray scales [22] as shown in figure 12. Other digital modulators use pulse width modulation (PWM) to achieve gray scales which directly erodes the data throughput of the devices. But GLVs, instead of using PWM to achieve gray scales, can be actuated to reach a defined displacement corresponding to a certain intensity level making these devices perfectly suitable for high speed applications. By increasing the resolution of the driving voltage, the GLVs can be easily programmed to different intensity levels extending the bit depth of the system. 8 bit or 10 bit grayscale GLVs [23] have already been reported and a bit depth as high as 16 bit has been proposed for use in a display application [24].

\section{- Mechanical stoppers as a pull-in protection mechanism}

As discussed before, one of the stringent constraints behind the operation of GLVs, is avoiding the accidental pull-in of the microbeams when $h_{0} / 3 \sim \lambda / 4$. Since the GLV microbeams are always at a higher potential compared to the bottom electrode (grounded), snapping down of the microbeams due to pull-in gives rise to a short circuit, potentially damaging the device. Hence, to avoid the short circuit, we did a thicker SiGe electrode deposition (within the airgap portion) which is electrically isolated from the rest of the bottom electrode and connected to the same metal line as of the microbeams through vias. Therefore, the electrostatic force acting on the fixed-fixed microbeam is still present because the larger area thin electrode does not interact with the equipotential bumps. The cross-section schematic and the relevant dimensions are shown in figure 13. Once the pull-in occurs, the microbeams fall on the thicker equipotential bumps instead of the grounded electrode avoiding the short circuit. Given the small contact area between the GLV beams and the bumps, the problem of stiction after pull-in is avoided. 
Figure 14 demonstrates the successful implementation of our pull-in protection mechanism. We used a $100 \mu \mathrm{m}$ long GLV microbeam having a pull-in voltage $\left(V_{P}\right)$ of $5.5 \mathrm{~V}$. As observed from the figure, for a triangular pulse pattern of max. $\pm 6.5 \mathrm{~V}$, the beam snaps down once $V_{P}$ is exceeded. After snapping on the thicker bumps, due to stationary nature of the microbeams, no further displacement is measured and hence a flat response is obtained. Soon after the triangular pulse strength reduces again below $V_{P}$, the movement of the microbeam is resumed and the displacements can be traced back again. No failure for the device was observed over several periods of operation which proves the usefulness of the stopper mechanism.

\section{Conclusion:}

We showed successful implementation of poly-SiGe as a MEMS material in fabricating GLVs. We described the fabrication procedure of our poly-SiGe GLV microbeams in detail and also indicated how it doesn't affect the underlying CMOS performance. The devices showed excellent behavior both for the static and dynamic responses. A contrast of more than 1500:1 was obtained with high diffraction efficiency. Since we operated with relatively short GLVs, the maximum diffracted light intensity was found to be strongly dependent on the length of the microbeams. The settling time of the devices was mainly dominated by the resonance frequency and the damping factor. Different dimensional parameters were varied to find their influence on the damping and hence the settling of the devices following a step function excitation. A minimum settling time of $2.2 \mu \mathrm{s}$ and $2 \mu \mathrm{s}$ were obtained for the $50 \mu \mathrm{m}$ and $75 \mu \mathrm{m}$ long devices showing under-damped and critically damped behavior respectively. We discussed how other system related trade-offs have to be managed once the dimensional parameters are varied to obtain faster switching. We proposed the use of mechanical stoppers to save the devices from erroneous deterioration due to pull-in. Overall these results prove the usefulness of the thin poly-SiGe MEMS technology for fabricating MOEMS devices directly on top of CMOS.

\section{Acknowledgement:}

We would like to thank the Flemish agency for Innovation through Science and Technology to support this work through the SBO Gemini project.

\section{References:}

[1] D. M. Bloom, The Grating Light Valve: Revolutionizing Display Technology, in: SPIE Proceedings of Projection Displays III Symposium 3013, 1997, pp. 165-171.

[2] J. I. Trisnadi, C. B. Carlisle and R. Monteverde, Overview and application of Grating Light Valve based optical write engines for high-speed digital imaging, in: Proceedings of Photonics West, Micromachining and Microfabrication symposium, (2004), Paper 5348-05.

[3] S. Rudra, J. Roels, G. Bryce, L. Haspeslagh, A. Witvrouw, D. Van Thourhout, SiGe based grating light valves: A leap towards monolithic integration of MOEMS, Microelectronic Engineering 87 (2010) 1195-1197.

[4] R. Jablonski, M. Turkowski and R. Szewczyk, Recent Advances in Mechatronics, Springer, 2007.

[5] A. Witvrouw, CMOS-MEMS integration today and tomorrow, Scripta Materialia 59 (2008), 945949.

[6] S. Sedky, A. Witvrouw, K. Baert, Poly-SiGe, a promising material for MEMS monolithic integration with the driving electronics, Sensors and Actuators A 97-98 (2002) 503-511. 
[7] L. Haspeslagh, J. De Coster, O.V. Pedreira, I. De Wolf, B. Du Bois, A. Verbist, R. Van Hoof, M.Willegems, S. Locorotondo, G. Bryce, et al. Highly reliable CMOS-integrated 11MPixel SiGebased micro-mirror arrays for high end industrial applications, in: Proceedings of IEEE International Electron Devices Meeting, 2008, pp. 1-4.

[8] S. Beeby, G. Ensell, M. Kraft and N. White, MEMS Mechanical Sensors, Artech House, 2004.

[9] D. Peroulis, S. P. Pacheco, K. Sarabandi and L. P. B. Katehi, Electromechanical considerations in developing low-voltage RF MEMS switches, IEEE Transactions on Microwave Theory and Techniques 51 (2003) 259-270.

[10] J.W. Goodman. Introduction to Fourier optics, third ed., Roberts \& Company Publishers, 2005.

[11] D. C. O'Shea, T. J. Suleski, A. D. Kathman and D. W. Prather, Diffractive optics: Design, Fabrication, and Test, SPIE Press Book, 2003.

[12] G.M. Rebeiz, RF MEMS: Theory, Design, and Technology, John Wiley \& Sons, 2003.

[13] H. A. C. Tilmans, M. Elwenspoek and J. H. J. Fluitman, Micro resonant force gauges, Sensors and Actuators A 30 (1992) 35-53.

[14] M. Bao, Analysis and design principles of MEMS devices, Elsevier, 2005.

[15] M. Bao and H. Yang, Squeeze film air damping in MEMS, Sensors and Actuators A 136 (2007) 3-27.

[16] H. Sumali, Squeeze-film damping in the free molecular regime: model validation and measurement on a MEMS, J. Micromech. Microeng. 17 (2007) 2231-2240.

[17] E. P. Furlani, Theory and simulation of viscous damped reflection phase gratings, J. Phys. D: App. Phys. 32 (1999) 412-416.

[18] G. Bryce, S. Severi, B. du Bois, M. Willegems, G. Claes, R. Van Hoof, L. Haspeslagh, S. Decoutere and A. Witvrouw, Simultaneous optimization of the material properties, uniformity and deposition rate of polycrystalline CVD and PECVD Silicon-Germanium layers for MEMS applications, in: Proc. ECS '08, ECS Transactions, 16(10), 2008, pp. 353-364.

[19] P. Gonzalez, B. Guo, M. Rakowski, K. De Meyer and A. Witvrouw, CMOS compatible polycrystalline silicon-germanium based pressure sensors, Sens. Actuators A: Phys. (2011), doi: 10.1016/j.sna.2011.12.018

[20] R. Beernaert, A. Avci, J. De Smet, H. De Smet, J. De Coster, S. Severi, and Ann Witvrouw, Novel analog pulse-width-modulated 15- $\mu \mathrm{m}$ SiGe micromirrors, J. Soc. Inf. Display 18 (2010) 855-861.

[21] S. P. Levitan, T. P. Kurzweg and M. Bails, System simulation of mixed-signal multi-domain microsystems with piecewise linear models, IEEE Transactions on Computer-Aided Design of Integrated Circuits and Systems 22 (2003) 139-154.

[22] D. T. Amm and R. W. Corrigan, Grating Light Valve ${ }^{T M}$ Technology: Update and Novel Applications, in: SID Symposium Digest of Technical Papers 29, 1998, pp. 29-32

[23] E. Tamaki, Y. Hashimoto, O. Leung, Computer-to-plate printing using the Grating Light Valve device in: Proceedings of Photonics West, Micromachining and Microfabrication symposium, (2004), Paper 5348-08.

[24] B. Winkler, D. Elkins, A. Tanner, D. Bloom, R. Yeh, V. Ramsey, R. Cuff, An optical microsystem for displays, in: Technical digest of the 2006 Solid State Sensors, Actuators and Microsystems workshop, 2006, pp. 15-18.

\section{Figure Captions:}

Figure 1: Process flow: (a) Deposition of oxide and underlying metal layer patterning, (b) adding the barrier layer and opening the via holes, (c) deposition of the electrode layer and patterning the oxide hardmask on top of it, (d) deposition of a thicker SiGe layer and patterning of the thin electrode (oxide hardmask) and thick electrode (resist mask) layer with a single etch, (e) deposition of a HDP Si-oxide and 
subsequent planarization of the electrodes by CMP, (f) deposition and patterning of the sacrificial oxide, (g) adding the anchors and the structural layer, (h) deposition and patterning of the bondpad metal, deposition of a protecting Si-oxide layer and patterning the structural layer, (i) final step to release the MEMS structure by removal of the sacrificial oxide.

Figure 2: SEM cross-section of the poly-SiGe structural layer and the SiC/Al bilayer on top of it.

Figure 3: Microscopic view of the GLV device consisting of an array of $50 \mu \mathrm{m}$ long fixed-fixed beams.

Figure 4: Observed variation in the resonance frequency with the length of the microbeams.

Figure 5: Schematic of the optical set-up used to characterize the contrast of the devices.

Figure 6: Analog response of GLV devices showing an excellent optical response.

Figure 7: Comparison of deflection of the microbeams and the Gaussian intensity distribution of the focused spot at the centre of the devices.

Figure 8: Variation in settling time of the devices with change in thickness of the air-gap.

Figure 9: Variation in settling time of the devices with change in width of the microbeams.

Figure 10: Variation in settling time of the devices with change in thickness of the microbeams.

Figure 11: Variation in settling time of the devices with change in length of microbeams.

Figure 12: Response of a GLV to a square wave pulse train showing the inherent analog nature of the device.

Figure 13: Design details of the distribution of equipotential thicker bumps and grounded thinner electrodes defining the pull-in protection mechanism.

Figure 14: Displacement of a pull-in protected GLV device in response to a triangular pulse pattern. 

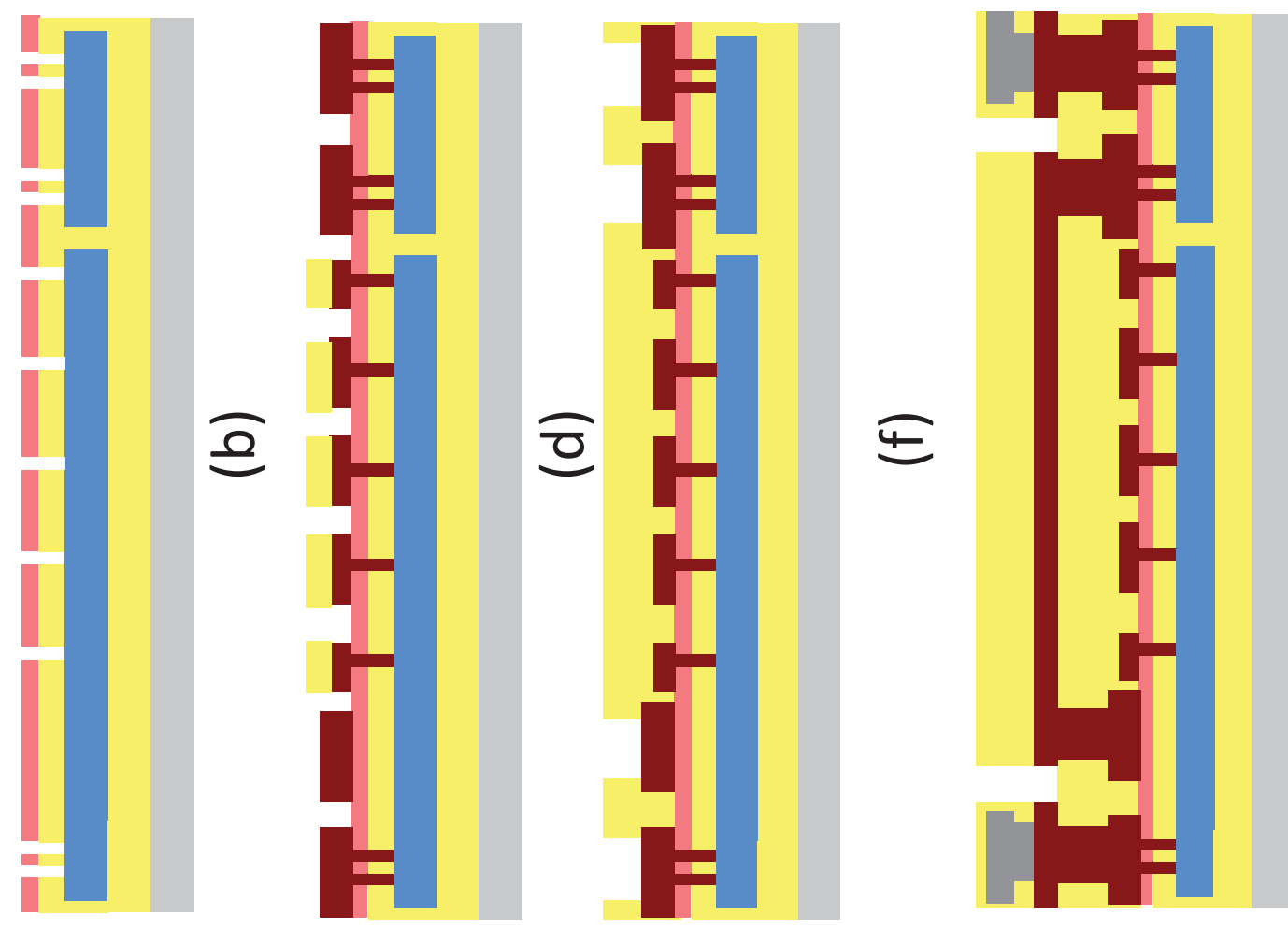

ఏ

过

$\square$

$\frac{\cup}{n}$

$\bar{r}$

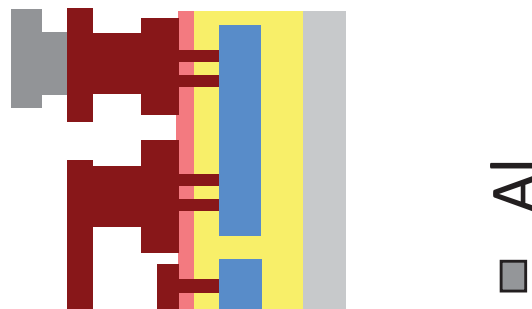

$=\underset{j}{z}$
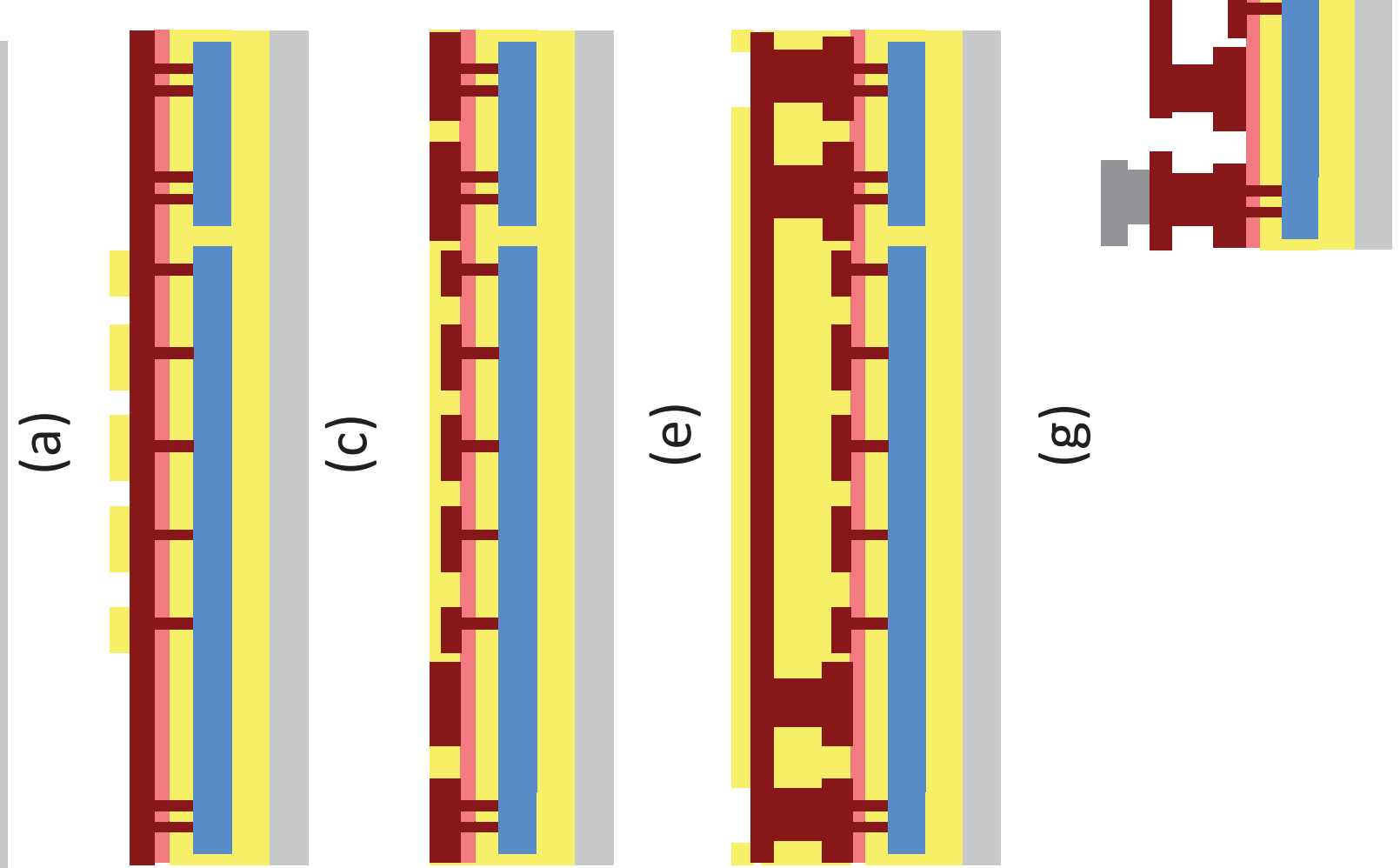

$\widetilde{00}$

ำ

(1)

옴

$\square$

is

$\square$

흘 


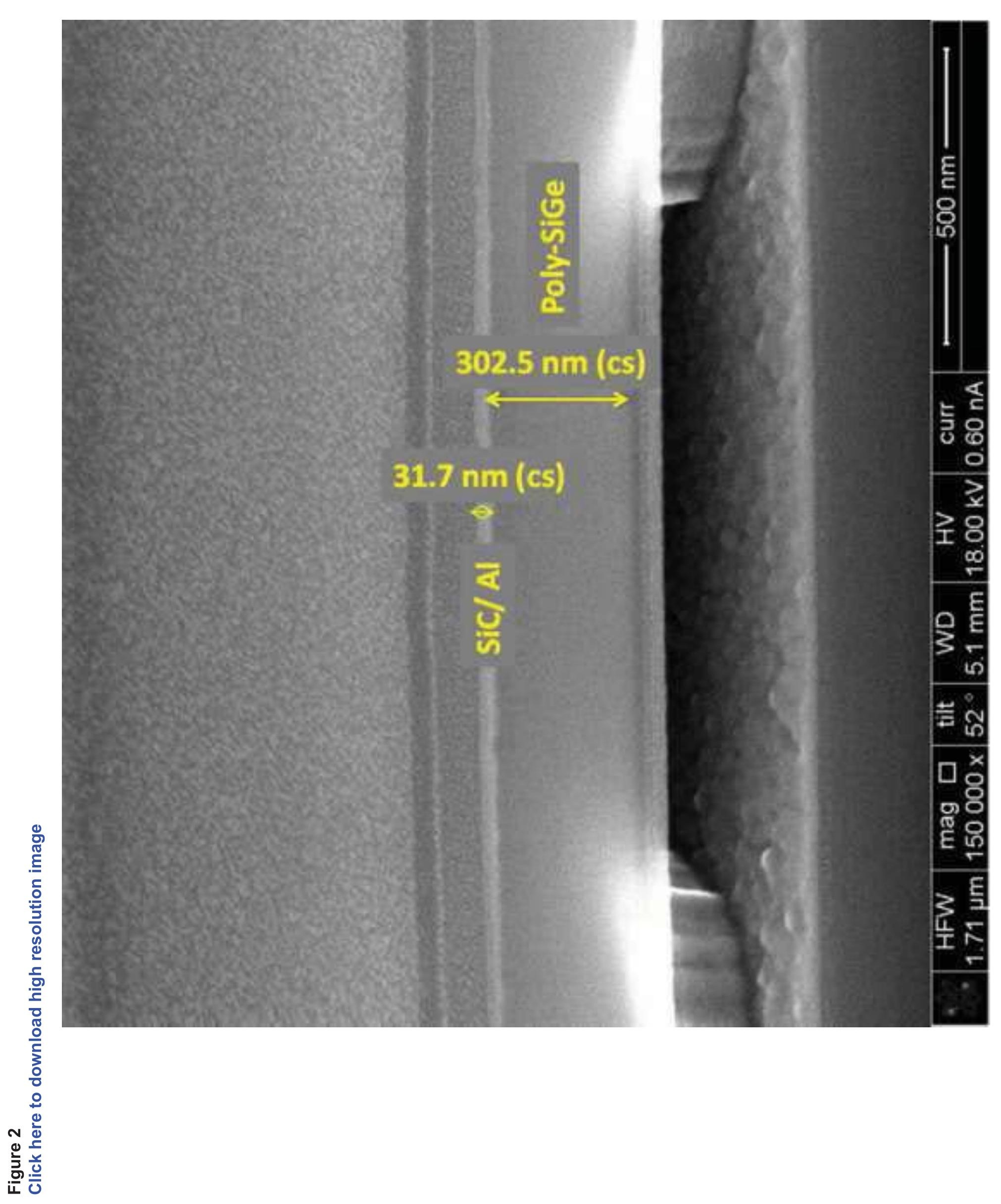




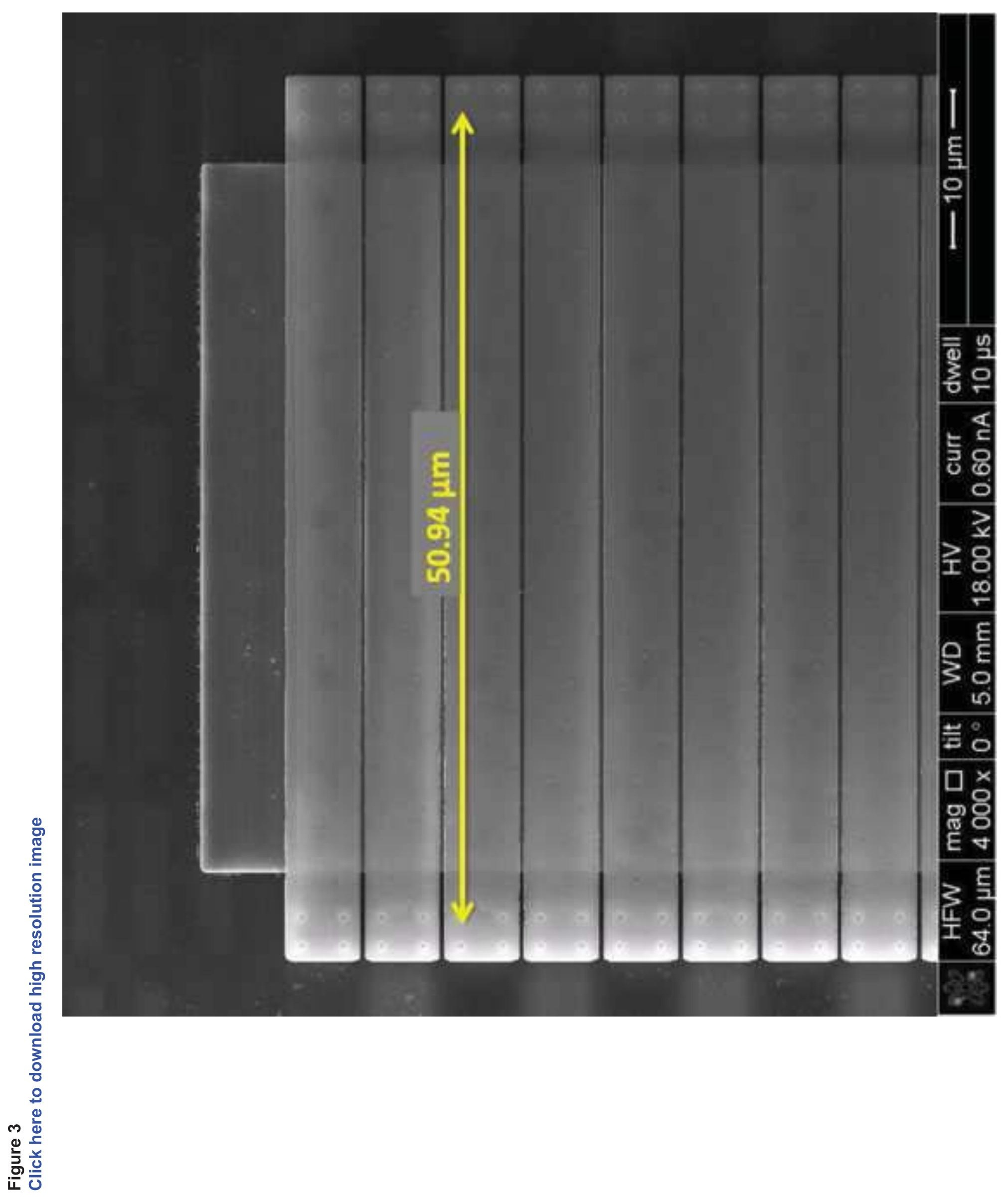




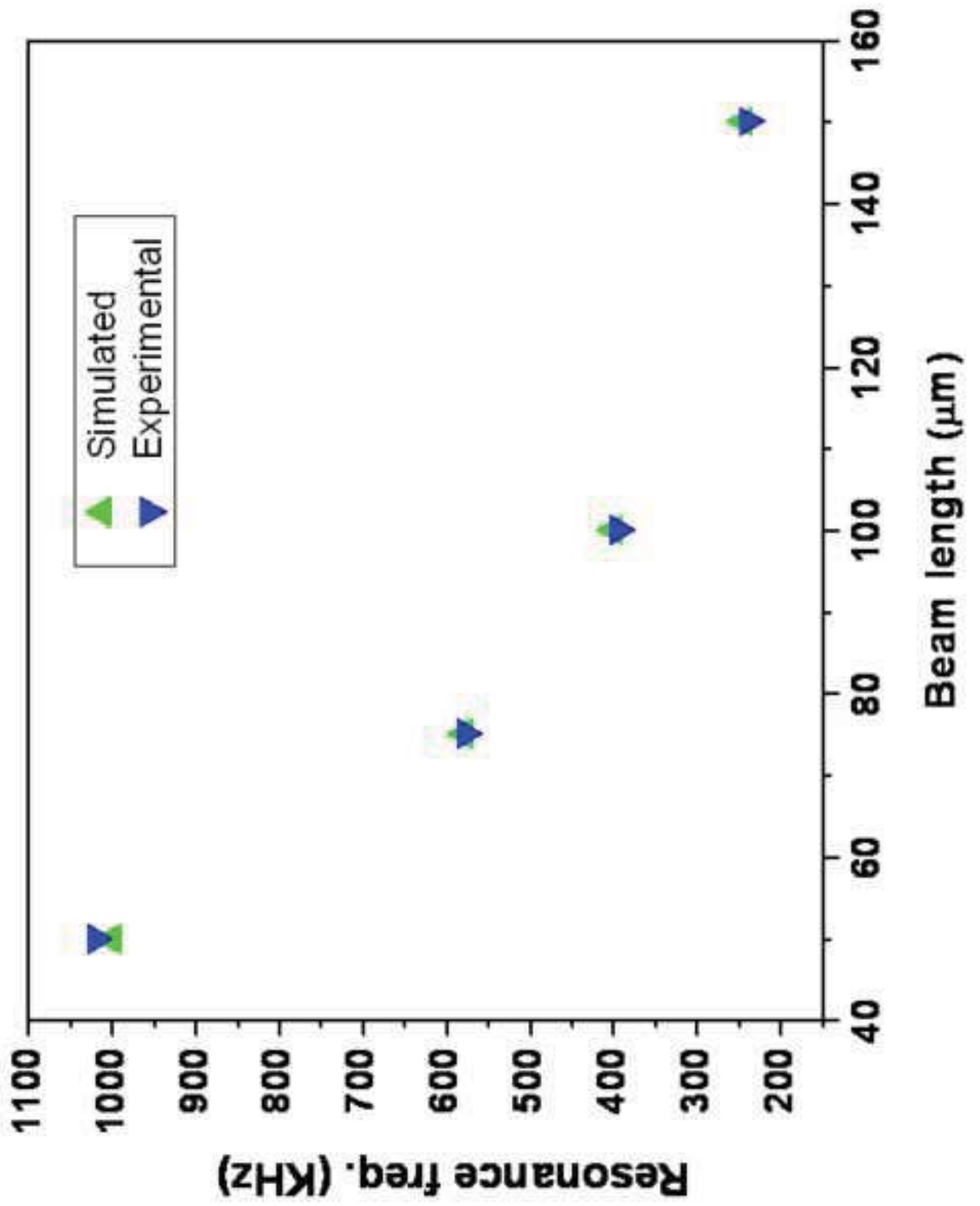




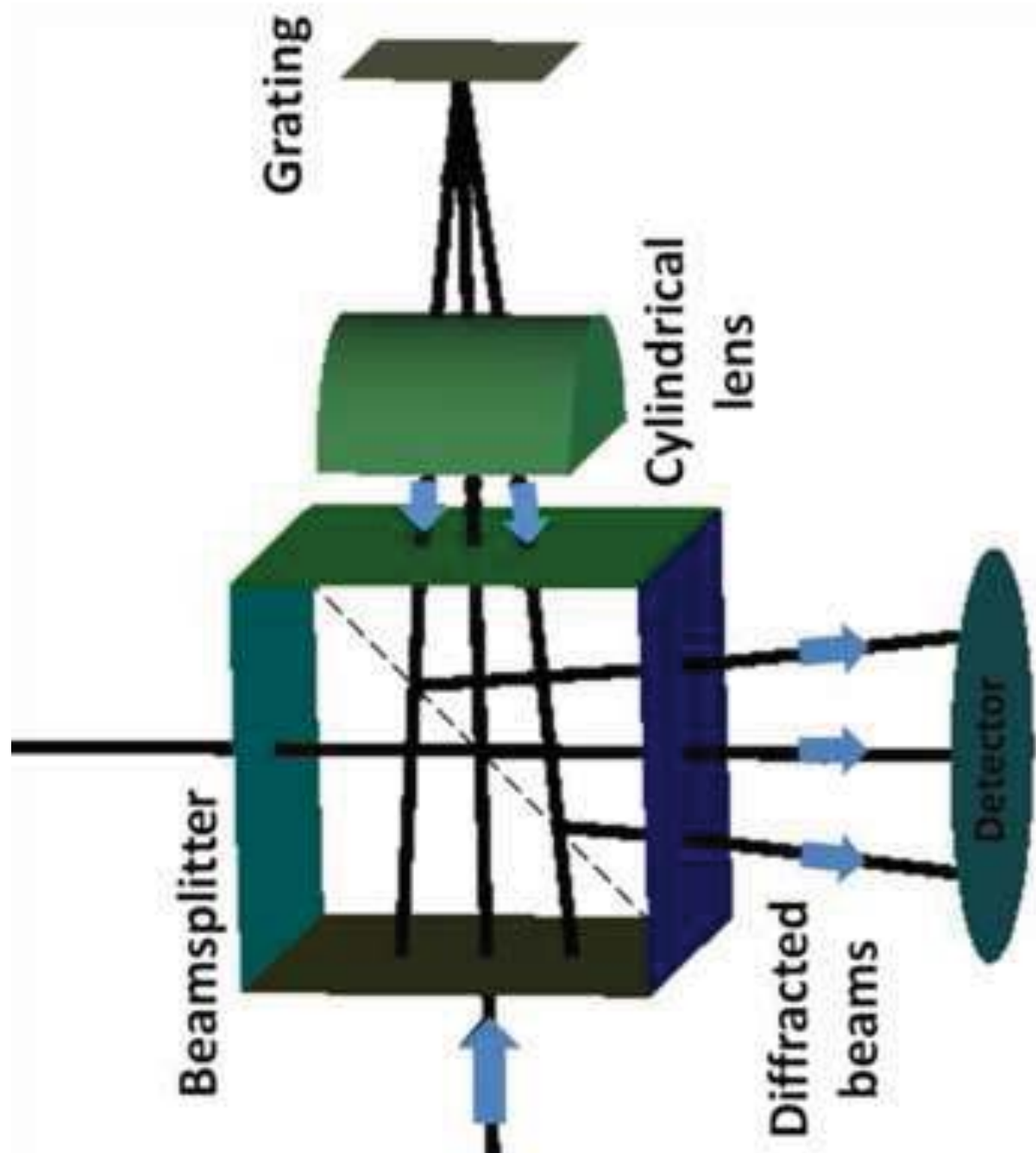

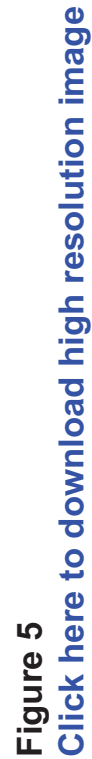




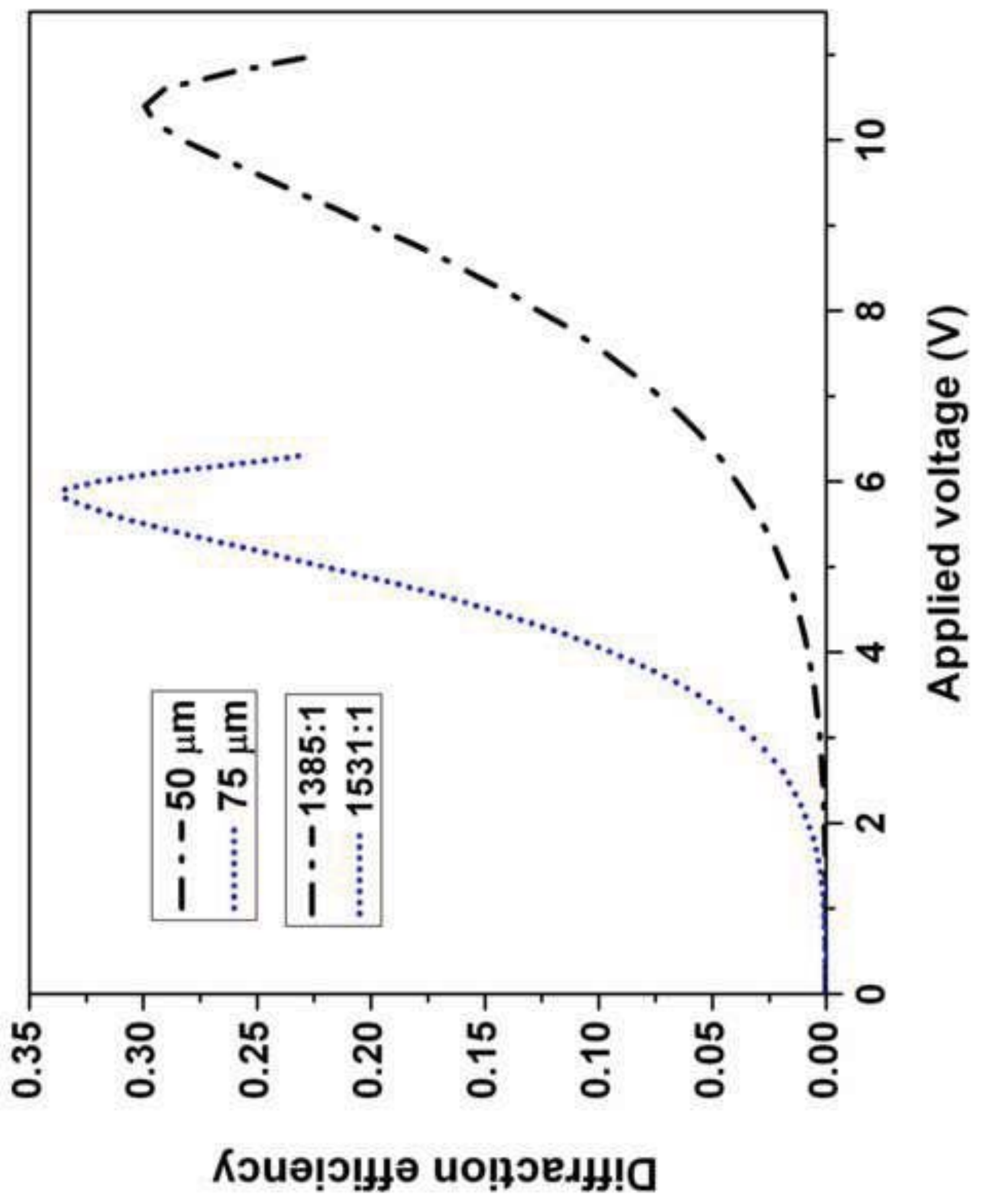




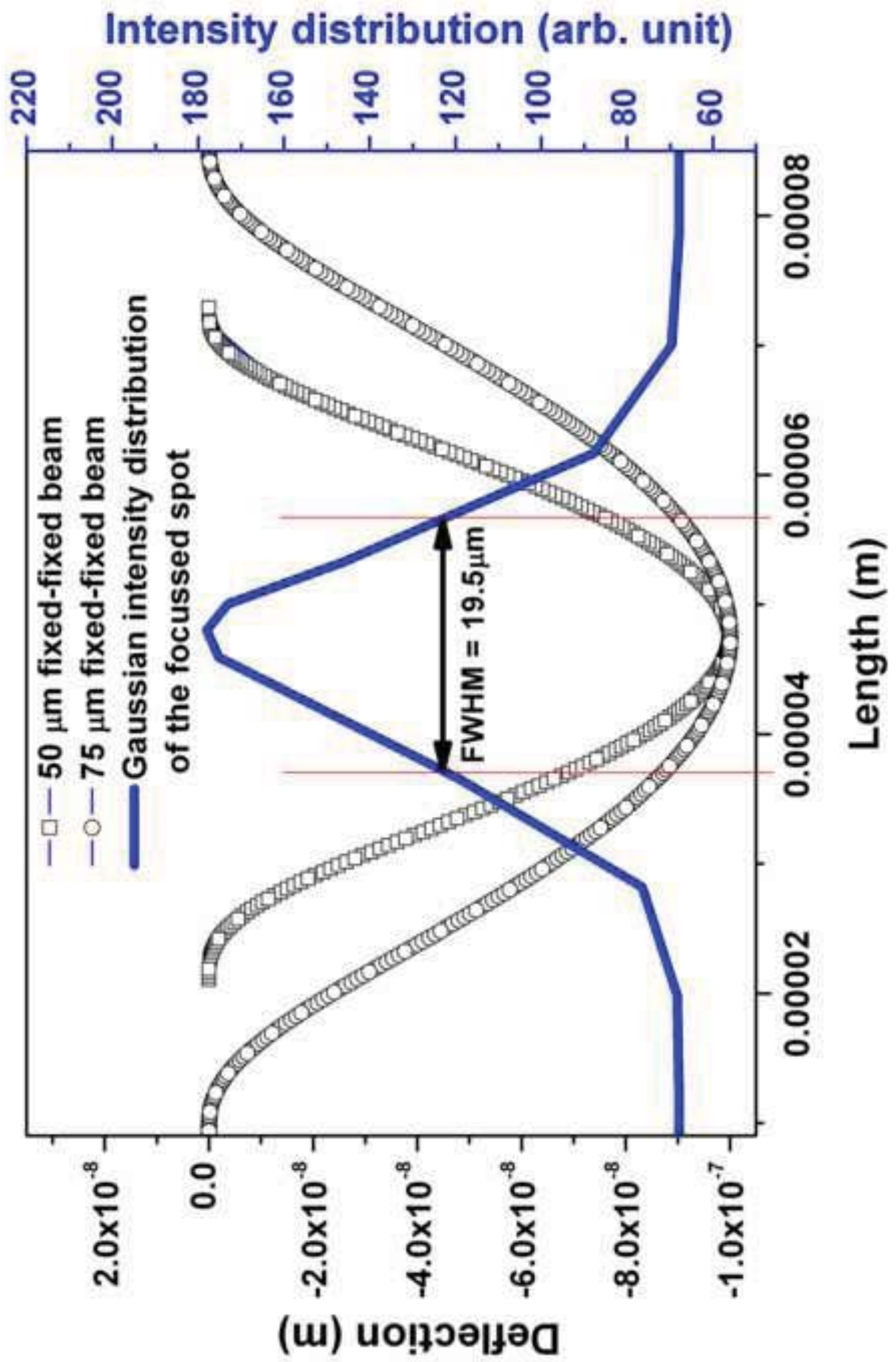




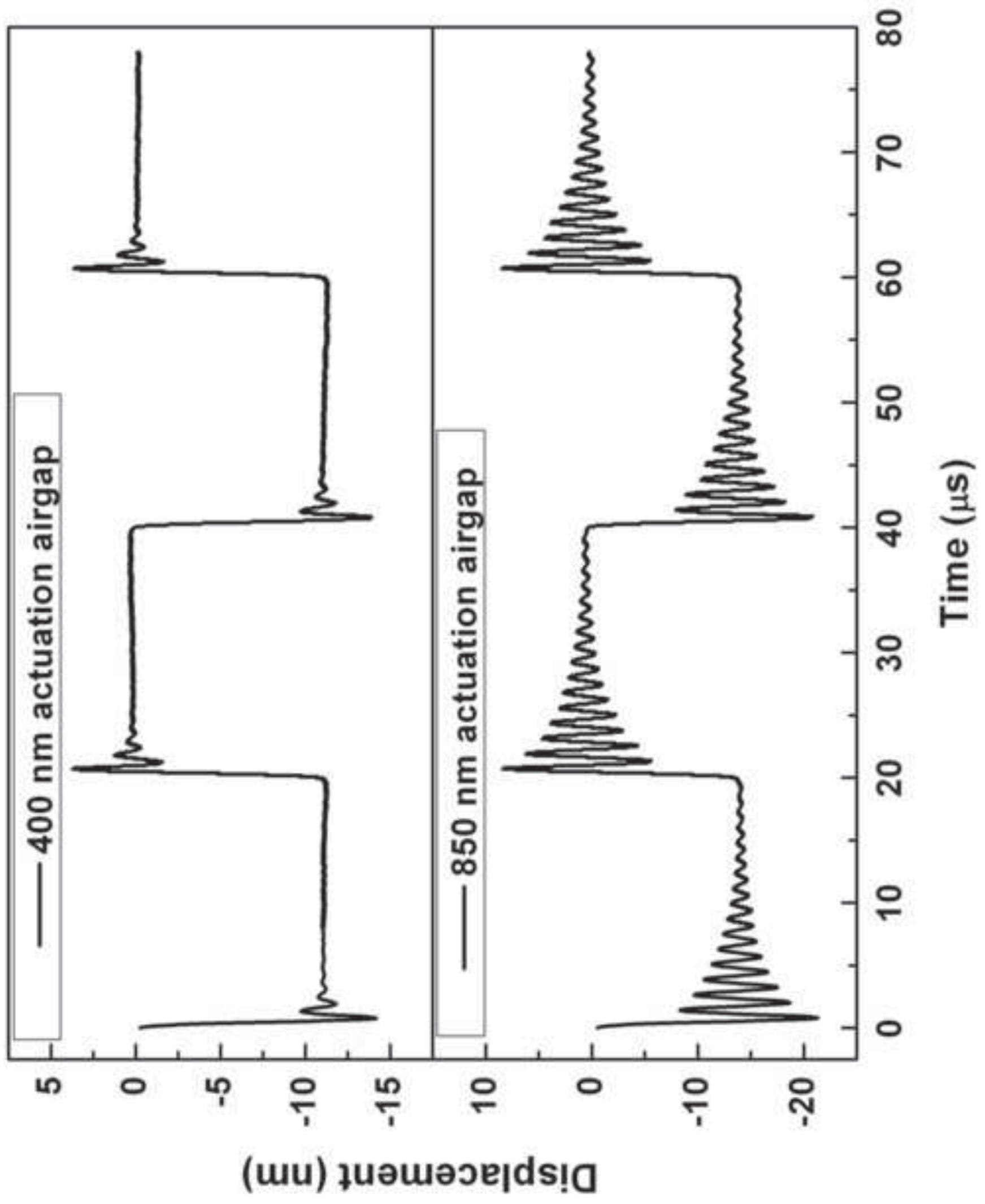




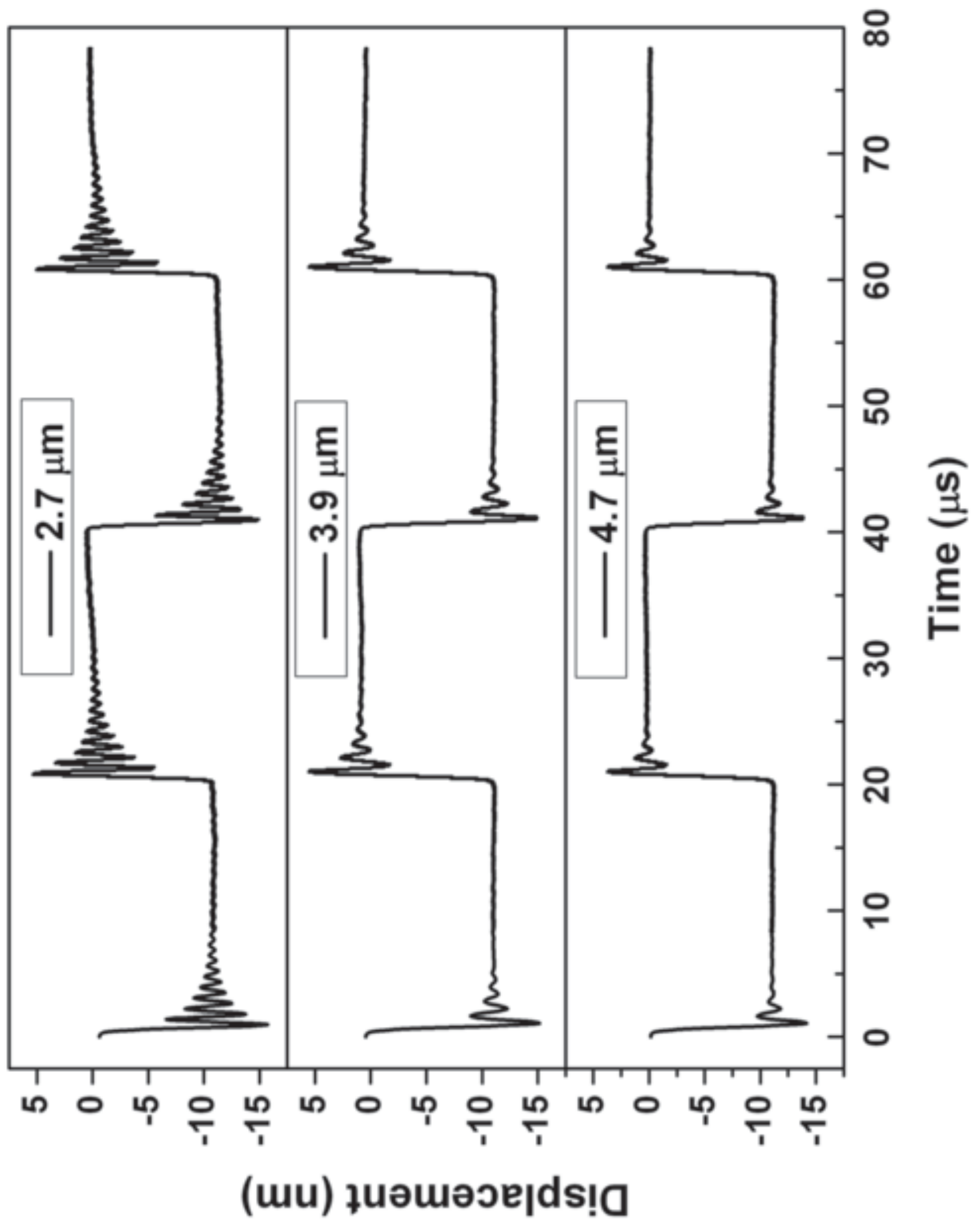




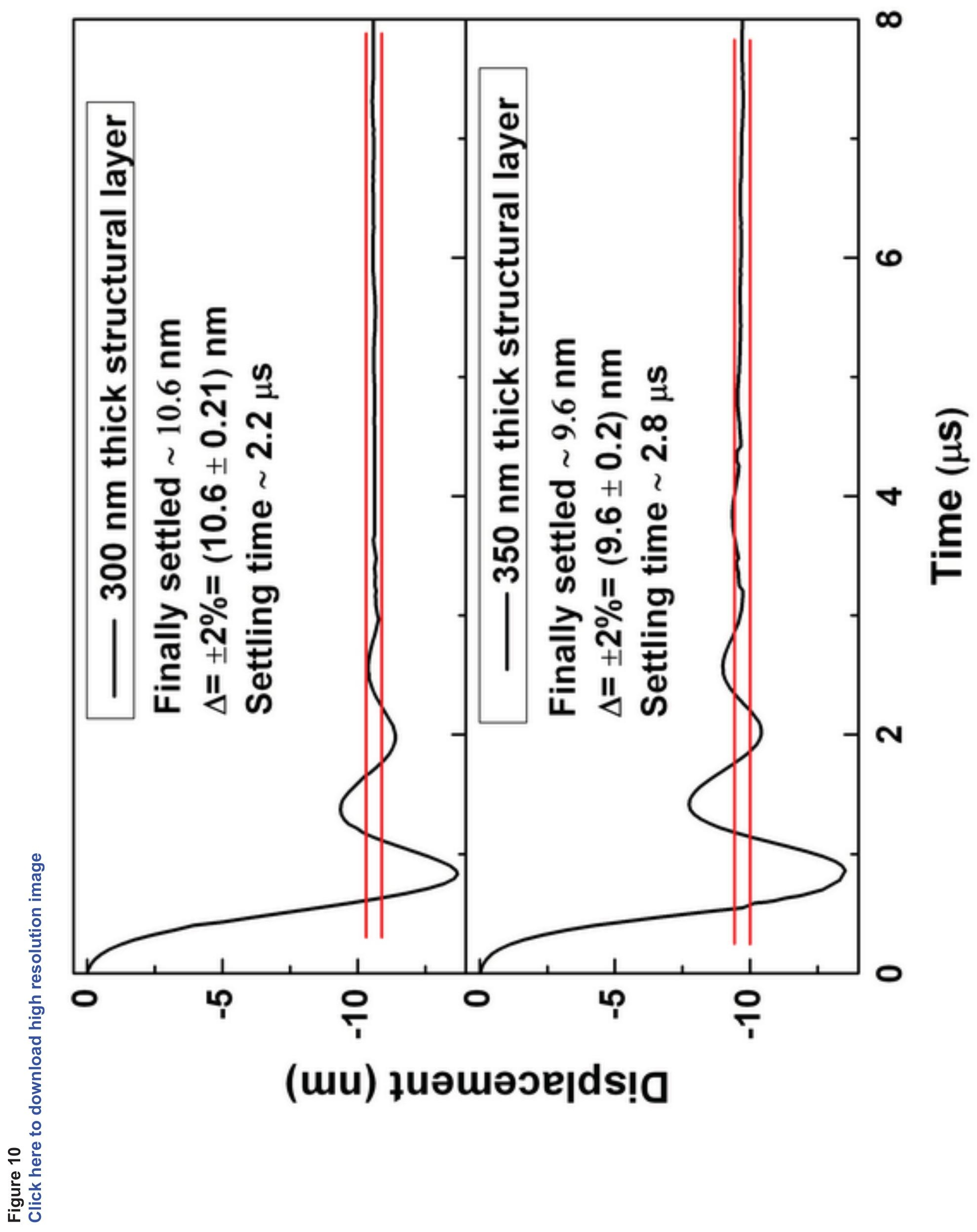




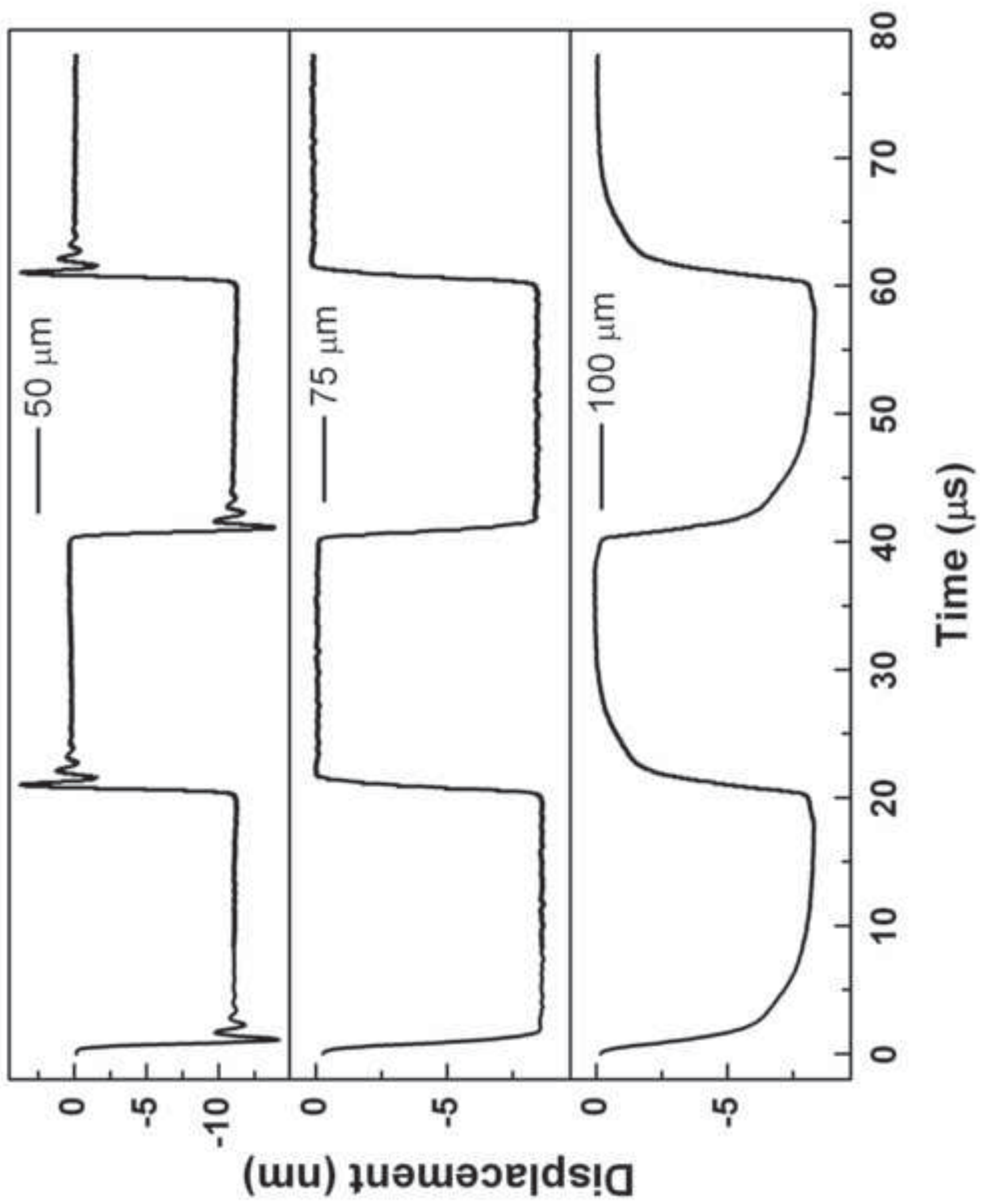




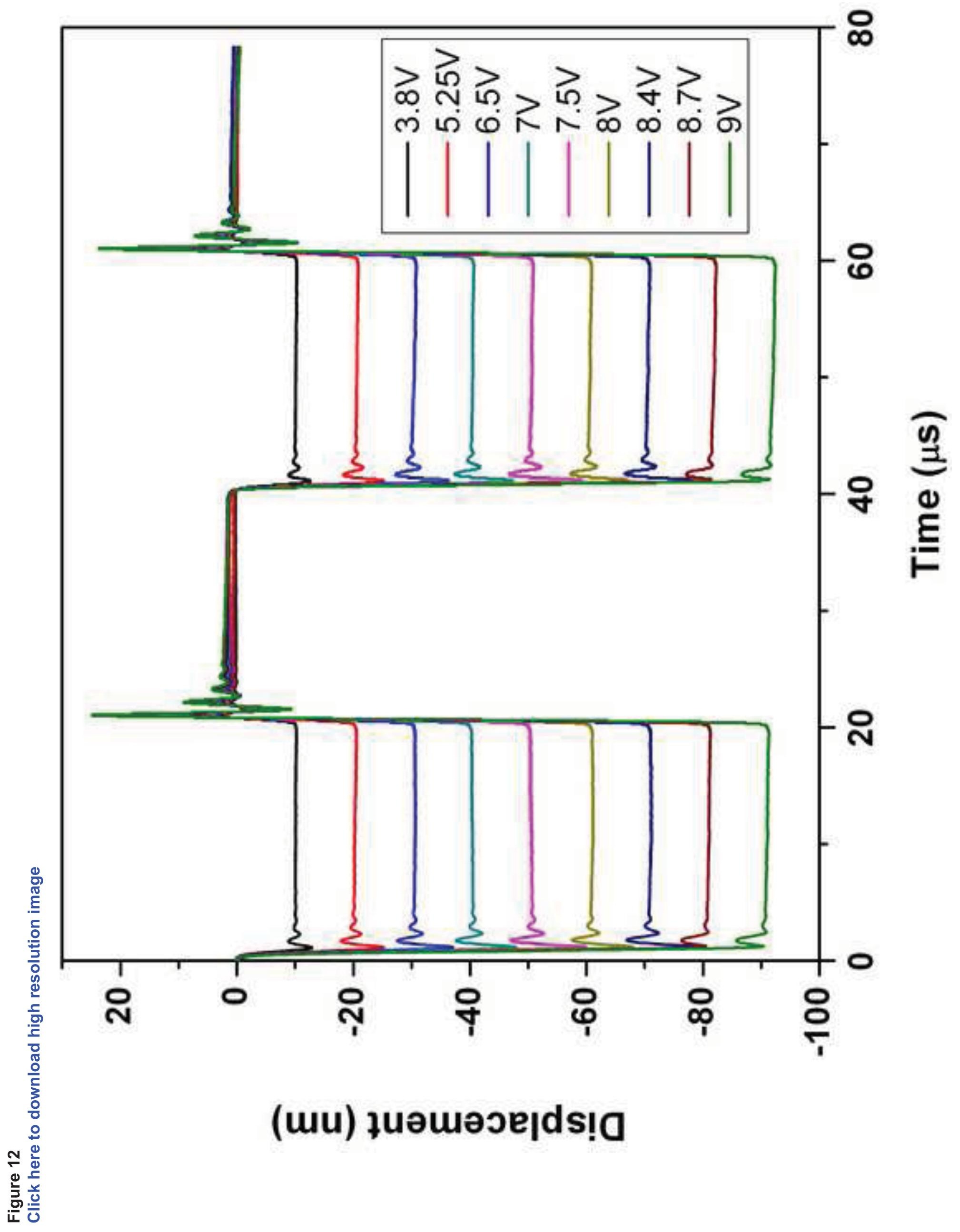




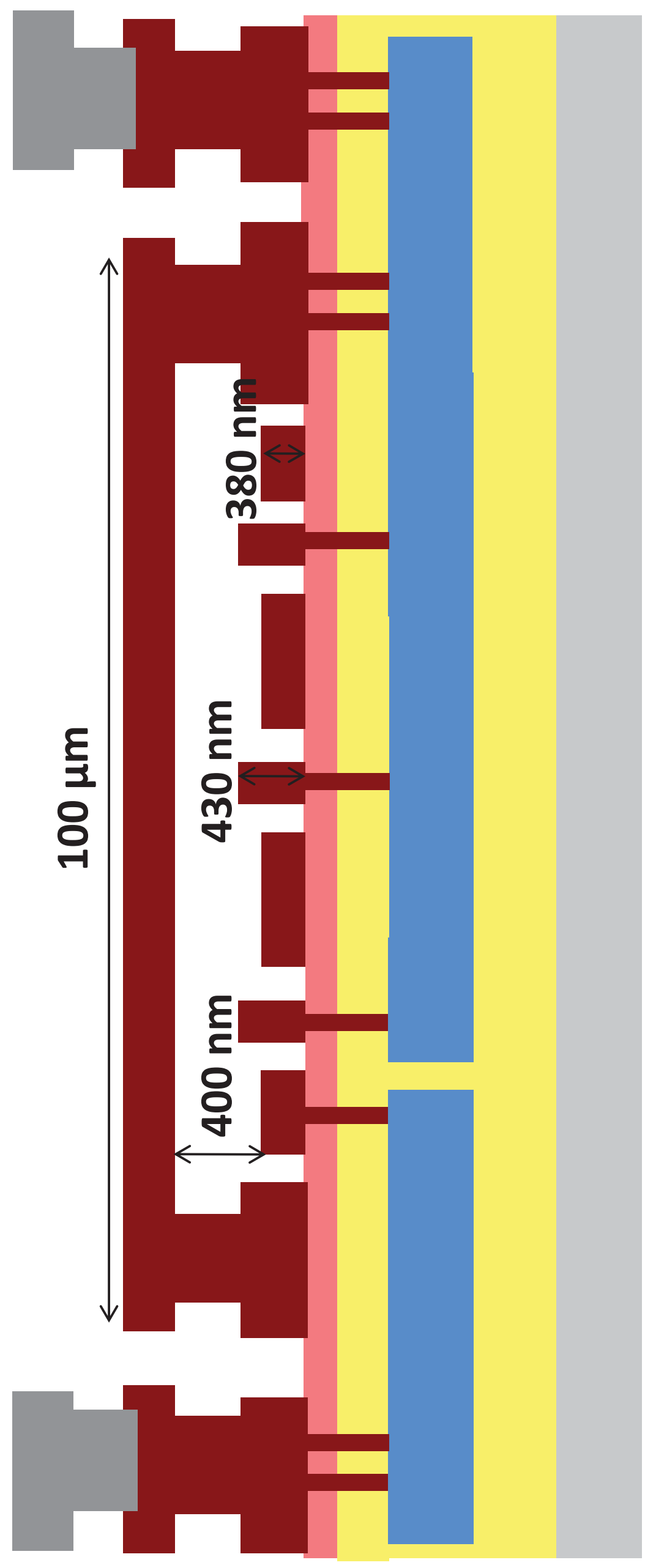

$\frac{m}{0}$
$\frac{0}{5}$
$\frac{0}{2}$ 


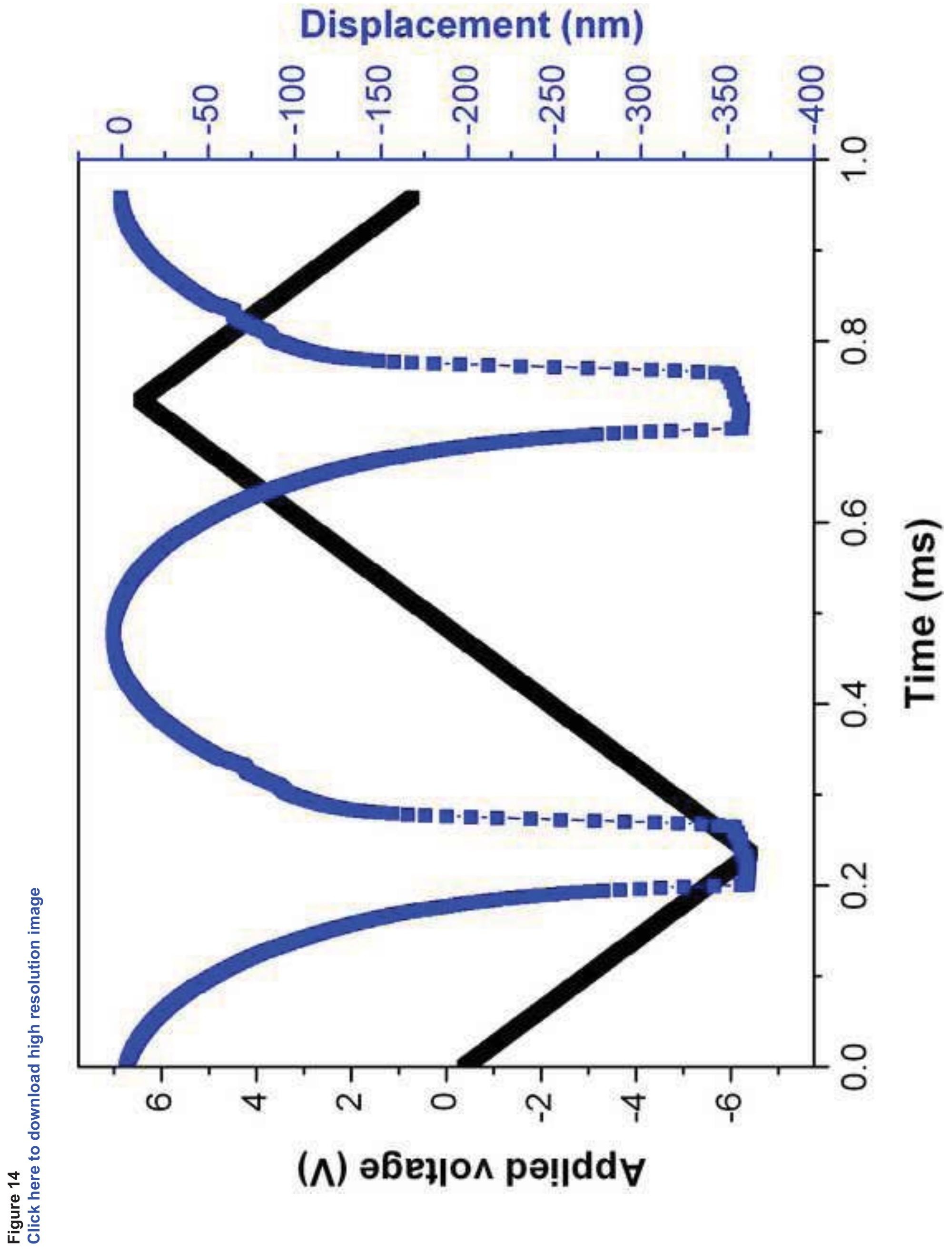

